\title{
1 Gd matrix effects on Eu isotope fractionation in geological rocks using MC- 2 ICP-MS: Optimizing Europium isotope ratio measurements in geological 3 samples
}

4

5

Seung-Gu Lee ${ }^{1}$, Tsuyoshi Tanaka²

6

$7^{1}$ Geology Division, Korea Institute of Geoscience and Mineral Resources, Daejeon 34132,

8 Korea

9²Institute for Space-Earth Environmental Research, Nagoya University, Nagoya 464-8601, 10 Japan

11

12Abstract

13Eu has only two isotopes $\left({ }^{151} \mathrm{Eu}\right.$ and $\left.{ }^{153} \mathrm{Eu}\right)$. Eu and Gd are one of the rare earth elements that 14are very difficult to completely separate from each other. Eu isotope ratio can be determined 15by MC-ICP-MS using internal Sm or Gd spikes to correct for mass discrimination. 16NIST3117a ultrapure chemical reagent shows almost no Eu isotope fractionation regardless 17 of the kind of normalization isotope pair. However, Eu isotope ratio in the silicate rocks was 18effected by Gd matrix during MC-ICP-MS measurement if a trace amount of Gd impurity 19remains in the purified Eu fraction. In this report, we tried to determine optimizing conditions 20for precise and accurate Europium isotope ratio measurements in geological samples using 21MC-ICP-MS. The pure Eu fraction with almost no Gd matrix separated from geological 22samples and NIST3117a ultrapure chemical reagent show almost same degree of Eu isotope 23 fractionation regardless of the kind of normalization isotope pair. However, Eu isotope ratio 24in the silicate rocks was effected by Gd matrix during MC-ICP-MS measurement using if $25^{154} \mathrm{Gd}$ interference relative to ${ }^{154} \mathrm{Sm}$ as internal standard is more than ca. $0.1 \%$. Particularly, 26highly fractionated granite and high silica volcanic rock with extremely low Eu concentration 27compared to Gd require high -purity Eu separation with a high recovery rate to obtain the 28true value of the Eu isotope fractionation in the geological rocks.

29Key words: Geological rocks, Eu isotope fractionation, Sm and Gd internal standard, Gd 30matrix, MC-ICP-MS

31Corresponding author: sgl@kigam.re.kr 


\section{Introduction}

33

34Abundances and isotopic ratios of REEs provides valuable information to the evolution of 35Earth and other planetary systems. Among the REEs, Eu holds considerable potential as a 36geochemical tool because it can assume both divalent (+II) and trivalent (+III) oxidation 37states. Most rare earth elements exist only in the trivalent state (+III) in nature. The divalent 380xidation state of Eu may cause elemental fractionation during magmatic evolution and may 39specifically indicate oxygen fugacity of the magmatic system [1-2]. Dauphas et al. [3] 40proposed a possibility of Eu isotope variation in bulk rocks and individual minerals due to 41redox condition in the mantles of Earth and other planets. Therefore, precise and accurate 42determination of Eu isotope ratio in the geological materials is challenging subject to 43demonstrate Eu isotope fractionation during the evolution of the Earth and solar system.

44The development of MC-ICP-MS methodologies, which can precisely and accurately 45measure isotopic ratios for a broad range of elements, has allowed for more detailed 46investigation of different geochemical and cosmochemical proxies. Since Moynier et al. [4] 47reported Eu isotope ratio from some meteorites, several researchers have developed analytical 48methods to measure stable isotope ratios of rare earth elements [4-11] using MC-ICP-MS and 49TIMS. Moynier et al. [4] had reported Eu isotope ratios in chondritic and environmental 50materials determined using a Sm isotope pair $\left({ }^{147} \mathrm{Sm}^{-}{ }^{149} \mathrm{Sm}\right.$ spike $)$ as an internal standard. Eu 51isotope ratio can be determined by SSB (standard-sample-bracketing, [11]) or C-SSBIN 52(Combined Standard-Sample-Bracketing Internal Normalization, [4, 9, 10]) method. 53Particularly, Lee and Tanaka [10] reported a method to determine Eu isotope ratio of high54purity commercial Eu reagents precisely and accurately by MC-ICP-MS using Sm internal 55standard. The authors also determined $\mathrm{Eu}$ isotope ratios from some geochemical reference 56materials (Table 1). In Table 1, except JG1a, the value of $\delta^{151 / 153} \mathrm{Eu}_{\text {NIST3117a }}$ do not show large 57difference though the stability of the data is that C-SSBIN method is better than SSB method. 58However, JG1a seems to indicate that Eu isotope ratio varies depending on the measurement 59method (C-SSBIN and SSB).

60 Analysis of Eu isotopic values from geological or meteoritic materials requires 61purification of Eu to prevent interference by coexisting elements. Gadolinium (Gd) especially 62can interfere with detection of Sm peaks that serve as an internal standard during 63measurement. Li et al. [12] reported that the distribution coefficient of Gd and Eu between 
64HIBA and AG50W-X8 resin during column chromatography overlapped, suggesting that 65both elements should elute together during the HIBA chemistry. Lee and Tanaka [10] 66described Eu separation method from REEs using 2-hydroxyisobutyric acid (HIBA, 0.12M) 67 as an eluant. However, we recently noticed that Eu isotopic determination method by MC68ICP-MS using Sm isotope spikes developed by Lee and Tanaka [10] carry the risk of Gd 69matrix effects due to interference of ${ }^{154} \mathrm{Sm}$ and ${ }^{154} \mathrm{Gd}$ isobars in the high silica igneous ricks.

70 In this research, we checked Gd matrix effects during Eu isotope ratio determination by 71MC-ICP-MS using a Sm internal standard and suggest an optimal method for determining Eu 72isotope ratio from the geological rocks precisely and accurately. We used a commercial 73 geochemical reference materials to compare Eu isotopic ratio estimates normalized according 74to different ratio pairs, ${ }^{147} \mathrm{Sm}_{-}{ }^{149} \mathrm{Sm},{ }^{147} \mathrm{Sm}-{ }^{152} \mathrm{Sm},{ }^{147} \mathrm{Sm}-{ }^{154} \mathrm{Sm},{ }^{149} \mathrm{Sm}-{ }^{154} \mathrm{Sm},{ }^{150} \mathrm{Sm}-{ }^{154} \mathrm{Sm}$ and $75^{155} \mathrm{Gd}-{ }^{157} \mathrm{Gd}$. The objective was to identify an optimal normalizing pair to minimize ${ }^{154} \mathrm{Gd}$ 76interference with ${ }^{154} \mathrm{Sm}$ signals used to normalize Eu isotopic ratios. This research also found 77that Eu isotopic ratio estimates depend on thorough separation of Gd prior to mass spectral 78analysis.

79

\section{Experimental Procedures}

81

\subsection{Reagents and methods}

83

84 The procedures used here followed those published in Lee and Tanaka [10]. Sample 85solutions for Eu isotopic measurements by MC-ICP-MS consisted of $2 \% \mathrm{HNO}_{3}$ prepared 86from 60\% ultrapure $\mathrm{HNO}_{3}$ (Merck, Darmstadt, Germany) and deionized water (Milli-Q 87system, Millipore, Milford, USA). NIST3117a was used as an in-house standard solution for 88determining $\mathrm{Eu}$ isotope ratios from geochemical reference materials. An in-house Sm 89solution was prepared from ultrapure $\mathrm{Sm}_{2} \mathrm{O}_{3}$ produced by Alfa Aesar. This, along with 90NIST3118a (Lot: 992004), was used as an in-house Gd spike for normalizing Eu isotopic 91ratios. The aforementioned Gd standard reagent was also used to investigate ${ }^{154} \mathrm{Gd}$ isotope 92interference with the ${ }^{154} \mathrm{Sm}$ spike used to measure Eu isotope ratio by MC-ICP-MS.

\section{3}

\subsection{Geological samples}

96 This study used well-known geochemical reference rocks BCR2, BIR1a, AGV2 and GSP2 
97curated by the U.S. Geological Survey and JB3, JG1a and JGb2 curated by the Geological 98Survey of Japan the U.S. Geological Survey. These are three basaltic rocks (BCR2, BIR1a 99and JB3), two granitic rocks (GSP2, JG1a), one gabbro (JGb2) and andesite (AGV2). Sample 100digestion procedures followed those described in Lee et al. [13, 14]. Commercially available 101ultrapure $\mathrm{HF}, \mathrm{HNO}_{3}$ and $\mathrm{HCl}$ along with sub-boiled, high-purity $\mathrm{HClO}_{4}$ (Merck) were used 102throughout analytical procedures including sample dissolution steps. Samples consisted of 103100-200 mg of powder weighed and digested in PFA Savillex with a 2:1 mixture of 104concentrated $\mathrm{HF}$ and $\mathrm{HNO}_{3}$ at ca. $160^{\circ} \mathrm{C}$ for more than 48 hours. After addition of $0.1 \mathrm{ml}$ 105concentrated $\mathrm{HClO}_{4}$, the sample solutions were dried at ca. $180^{\circ} \mathrm{C}$ for more than a day. After 106drying, the cake was treated by aqua regia and dried again. Finally, the cakes were re107dissolved in $4 \mathrm{ml}$ of $2 \mathrm{M} \mathrm{HCl}$ for column chromatography. Before column chromatography, 108the solutions were checked for visual clarity to ensure total decomposition of the sample.

109

\subsection{Column chemistry for Eu separation}

111

112 Complete separation of Eu from other REEs is essential for precise determination of Eu 113isotopic ratios. Separation becomes even more critical for downstream analytical procedures 114that use neighboring elements (e.g., Sm and Gd) to constrain Eu concentrations. In this study, 115we used a quartz glass (ø $3 \mathrm{~mm}$, length $98 \mathrm{~mm}$ ) chromatographic column packed with $0.8 \mathrm{~mL}$ 116of a cation-exchange resin (Biorad AG 50WX-8, 200-400mesh) and 2-hydroxyisobutyric 117acid (HIBA, Tokyo Chemical Industry, Japan) as an eluant. This method by Lee and Tanaka 118[10] is optimized to achieve the best separation of Eu from other REEs and provides better Eu 119recovery than that provided by the previous methods of de Carvalho et al. [9].

120 Due to the importance of Eu separation in generating precise and accurate results, a 121detailed description of this part of the procedure is provided. Eu was separated from other 122elements using two-step cation exchange column chromatography. A Biorad AG50W X-8 123resin (200-400 mesh stationary phase) was used in the column. After loading $4 \mathrm{ml}$ of the 124sample onto the pre-cleaned column, matrix was removed with $40 \mathrm{~mL}$ of $2 \mathrm{~N} \mathrm{HCl}$. The REE 125 fraction was eluted from the resin using $30 \mathrm{~mL}$ of $6 \mathrm{~N} \mathrm{HCl}$. Next, the REE fraction was 126injected onto a quartz glass column (ø $3 \mathrm{~mm}$, length $98 \mathrm{~mm}$ ) filled with $0.8 \mathrm{~mL}$ of the cation127exchange resin (Biorad AG 50WX-8, 200-400mesh). Eu was separated using 2128hydroxyisobutyric acid (HIBA, $0.12 \mathrm{M}$ ) as an eluant (Fig. 1). The eluant came from a 2M 
129stock solution of HIBA adjusted to $\mathrm{pH} 4.55$ using ultrapure $\mathrm{NH}_{4} \mathrm{OH}$ (Tamapure chemicals, 130Kawasaki, Japan). Prior to drying of the eluted Eu fraction, its purity was checked by ICP131MS (NexION 350, Perkin Elmer) or MC-ICP-MS. After the purity check, the Eu solutions 132 were dried at $160^{\circ} \mathrm{C}$ and re-dissolved in a $2 \% \mathrm{HNO}_{3}$ solution containing $\mathrm{Sm}$ or Gd internal 133standard spikes for Eu isotope ratio measurement.

134

\subsection{Mass spectrometry}

$136 \mathrm{Eu}$ isotopic measurements were performed on a Neptune Plus MC-ICP-MS (Thermo 137Fisher Scientific Ltd.) with nine Faraday cups and operated under wet plasma conditions at 138the Korea Institute of Geoscience and Mineral Resources (KIGAM), Daejeon, Korea. An 139aliquot of the NIST3117a standard used by Lee and Tanaka [10] was used as a reference. 140Measurements were performed in low-resolution mode with the Ni normal- sampling cone 141 and Ni X-skimmer cones.

142 The typical sample aspiration rate was $80-100 \mu \mathrm{L} / \mathrm{min}$. The gain on each Faraday cup was 143monitored on a daily basis to ensure normalization of its efficiency. Table 2 summarize the 144cup configuration and other operating parameters of Neptune Plus MC-ICP-MS. Previous 145research efforts [10] used seven Faraday cups but this study used nine Faraday cups to $146 \mathrm{measure}{ }^{147} \mathrm{Sm}$ and ${ }^{157} \mathrm{Gd}$.

147 Each sample was subjected 50 cycles (50cycles/block) with a 4.19 s integration interval. 148Blanks were analyzed before and after each sample measurement.

1502.5. Use of Sm and Gd internal standards for measuring Eu isotopic ratios in geological 151 samples

152

153 Lee and Tanaka [10] used ${ }^{150} \mathrm{Sm}$ and ${ }^{154} \mathrm{Sm}$ as internal standard reference peaks for Eu 154isotope ratio measurements. Most geological or meteoritic samples contain Sm and Gd at 155concentrations about three times higher than those of Eu. Even though Eu analytes had been 156purified to $99.9 \%$, detectable fractions of Sm and Gd remained in samples probably due 157incomplete column chromatography. While they are expected even for state-of-the-art 158separation procedures (described above), Gd impurities in the matrix include ${ }^{154} \mathrm{Gd}$ which acts 159as an isobar to ${ }^{154} \mathrm{Sm}$ and therefore interferes with peaks needed to normalize Eu signal.

160 In this study, we systematically analyzed Gd interference with Sm spikes during Eu 
161isotopic ratio determination. We specifically investigated Eu measurement stability for Sm 162isotope pairs ${ }^{147} \mathrm{Sm}_{-}{ }^{149} \mathrm{Sm},{ }^{147} \mathrm{Sm}^{-152} \mathrm{Sm},{ }^{147} \mathrm{Sm}-{ }^{154} \mathrm{Sm},{ }^{149} \mathrm{Sm}^{-}{ }^{154} \mathrm{Sm}$ and ${ }^{150} \mathrm{Sm}-{ }^{154} \mathrm{Sm}$. We also 163measured Eu isotopic ratios using ${ }^{155} \mathrm{Gd}$ and ${ }^{157} \mathrm{Gd}$ as internal reference peaks to determine 164variation they may introduce to measurements.

165 Eu isotope ratios are reported in standard delta notation (\%o) relative to the NIST3117a Eu 166standard solution:

167

$168 \delta_{\square}^{\frac{151}{153}} E u_{\square}(\%)=i$

169 where $i$ and $i$ are the mass bias corrected ratios of the sample and NIST 3117a, 170respectively.

171 Using the methods described above, we examined the $\delta^{151 / 153} \mathrm{Eu}$ ratios for the NIST3117a 172 and the geological reference materials listed above.

173

1742.6. Mass-dependent isotopic fractionation

175

176 As with similar analytical techniques, internal isotopic fractionation, referred to as mass 177discrimination (i.e., mass bias) occurs during MC-ICP-MS analysis [15-20]. Mass 178discrimination can arise due to space charge effects in the plasma or from vacuum effects [6, 17917]. Lee and Tanaka [10] used an ${ }^{150} \mathrm{Sm}^{-154} \mathrm{Sm}$ isotope pair to correct for mass-discrimination 180effects among Eu isotopes during MC-ICP-MS analysis. The authors used because these 181nuclides at masses 150 to 154 bracket Eu’s own mass numbers of 151 and 153 . Approaches 182using different isotope pairs are described in Moynier et al. [4] and de Carvalho et al. [9]. All 183of these studies found consistently detectable degrees of $\mathrm{Eu}$ isotope fractionation in 184geological materials. However, normalization using a pair of isotope spikes (or a spike of 185isotope pairs) may suffer from unresolved peaks for the heavier isotope in the pair or isobaric 186interference with either isotope in the pair due to poor purification during sample preparation. 187Comparing results produced by different isotope pairs can help identify the pair that 188minimizes measurement uncertainties introduced by these effects.

189 This study compared and corrected mass-discrimination effects on Eu isotopes using an 190external correction technique that assumes an exponential relationship between unknowns 191 and the reference isotope pair $\left({ }^{147} \mathrm{Sm}_{-}{ }^{149} \mathrm{Sm},{ }^{147} \mathrm{Sm}^{-152} \mathrm{Sm},{ }^{147} \mathrm{Sm}-{ }^{154} \mathrm{Sm},{ }^{149} \mathrm{Sm}^{-154} \mathrm{Sm},{ }^{150} \mathrm{Sm}-\right.$ $192{ }^{154} \mathrm{Sm}$ and $\left.{ }^{155} \mathrm{Gd}-{ }^{157} \mathrm{Gd}\right)$. 
194 The correction was as follows:

195

$196\left({ }^{151} \mathrm{Eu} /{ }^{153} \mathrm{Eu}\right)_{\text {corr }}=$

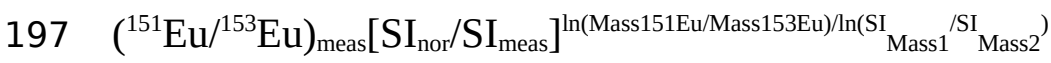

198(2)

199

200 where the subscripts 'meas' and 'corr' denote the measured and corrected isotope ratios, 201respectively. $\mathrm{SI}_{\text {nor }}$ and $\mathrm{SI}_{\text {meas }}$ indicate the normalization value and measured value of the spike 202isotope, respectively. The terms 'Mass151Eu', 'Mass153Eu' respectively refer to the atomic 203mass of ${ }^{151} \mathrm{Eu}$ and ${ }^{153} \mathrm{Eu}$, while ' $\mathrm{SI}_{\text {Mass1' }}$ ' and ' $\mathrm{SI}_{\text {Mass2 }}$ ' refer to lighter and heavier members of 204the spike isotope pairs, ${ }^{147} \mathrm{Sm}_{-}{ }^{149} \mathrm{Sm},{ }^{147} \mathrm{Sm}-{ }^{152} \mathrm{Sm},{ }^{147} \mathrm{Sm}_{-}{ }^{154} \mathrm{Sm},{ }^{149} \mathrm{Sm}^{-154} \mathrm{Sm},{ }^{150} \mathrm{Sm}^{-154} \mathrm{Sm}$ and $205^{155} \mathrm{Gd}-{ }^{157} \mathrm{Gd}$.

206 In a previous report [10], we assumed a natural abundance of ${ }^{150} \mathrm{Sm} /{ }^{154} \mathrm{Sm}=0.3244$ which 207represented a rounded average of values reported by Hidaka et al. $(0.324395,[21])$ and by 208Mass and McCulloch (0.324401, [22]). This study however used natural abundance ratios of 209samarium reported by Dubois et al. [23] to perform mass bias corrections. These values were 210determined by the total vaporization method using Johnson \& Matthey Sm oxides and as 211follows; ${ }^{147} \mathrm{Sm} /{ }^{154} \mathrm{Sm}=0.6626506,{ }^{147} \mathrm{Sm} /{ }^{149} \mathrm{Sm}=1.0868,{ }^{147} \mathrm{Sm} /{ }^{152} \mathrm{Sm}=0.562992$, $212^{149} \mathrm{Sm} /{ }^{154} \mathrm{Sm}=0.609726$ and ${ }^{150} \mathrm{Sm} /{ }^{154} \mathrm{Sm}=0.325396$. Previously assumed values of $213^{150} \mathrm{Sm} /{ }^{154} \mathrm{Sm}=0.3244$ [10] and ${ }^{155} \mathrm{Gd} /{ }^{157} \mathrm{Gd}=0.947645$ [23] also were used in spike isotope 214normalization for comparison. We could confirm that the calculated value of Eu isotope 215 fractionation $\left(\delta^{151 / 153} \mathrm{Eu}_{\text {NIST3117a }}\right)$ is the same regardless of the number $\left({ }^{150} \mathrm{Sm} /{ }^{154} \mathrm{Sm}=0.3244\right.$ or 2160.325396).

217 Elements in the sample matrix may result in doubly charged or polyatomic ions [24-29] 218that interfere with signals of interest. The mass range that includes ${ }^{151} \mathrm{Eu}$ and ${ }^{153} \mathrm{Eu}$ spans from $219^{150} \mathrm{Sm}$ to ${ }^{154} \mathrm{Sm}$ and includes both ${ }^{152} \mathrm{Sm}$ and ${ }^{152} \mathrm{Gd}$. This set of peaks makes Eu isotopes 220difficult to resolve and their proximity to the peaks of interest makes interfering peaks more 221difficult to isolate. In addition, ${ }^{150} \mathrm{SmH},{ }^{152} \mathrm{SmH}$ and ${ }^{152} \mathrm{GdH}$ can also interfere with ${ }^{151} \mathrm{Eu}$ and $222^{153} \mathrm{Eu}$ during MC-ICP-MS analysis. We did not detect this interference however during 223analysis of Eu isotopes from NIST3117a or an in-house Sm standard. 


\section{Results and Discussion}

2263.1. Variation in Eu isotope ratio estimates with different internal spike isotope pairs 227

228 Table 3 summarizes Eu isotope ratio estimates of NIST3117a for different Sm and Gd 229isotopic pairs used as an internal standard during MC-ICP-MS analysis. Table 4 and Fig. 3 230show clearly that, in NIST3117a Eu standard reagent, different Sm isotope pairs used for 231normalization give highly similar $\mathrm{Eu}$ isotope ratio estimates. These results suggest that 232ultrapure Eu fraction without Gd matrix provide the same value of Eu isotope ratio regardless 233the kind of Sm isotope pair for normalization. In addition, in Table 3 and Fig. 3, Eu isotope 234ratio normalized by $\mathrm{Gd}$ isotope pair $\left({ }^{155} \mathrm{Gd}-{ }^{157} \mathrm{Gd}\right)$ appeared to have more mass discrimination 235and instability compared to Sm normalization. However, because it appears to have a slight 236isotope fractionation of Eu isotopes, Eu isotope determination by Gd isotope normalization 237needs more study.

\section{8}

\subsection{Recovery rate of Eu, Sm and Gd in igneous rocks}

240 Though de Carvalho et al. [9] mentioned that Sm and Gd do not significantly affect Eu 241isotopic measurements given standard separation and analytical methods, their data (see 242Table 4 of [9]) showed slight, systematic variation of Eu isotope ratio (for example, -0.16 \%o 243to $0.02 \%$ for SSB methods and of $-0.09 \%$ to $0.04 \%$ for C-SSBIN methods with Gd 244concentrations ranging from 1.0 to $5.0 \mu \mathrm{g} / \mathrm{ml}$ ). Many studies have noted systematic isotopic 245bias introduced by incomplete separation [28, 29]. Lee and Tanaka [10] also emphasized the 246importance of complete separation of Eu from Sm and Gd when these latter elements are 247used as spikes. However, Lee and Tanaka [10] did not establish the degree of Eu isotope 248fractionation introduced by Gd matrix with Sm normalization or by Sm matrix with Gd 249normalization. This study estimated the extent to which incomplete separation of Sm, Eu and 250Gd influences Eu isotope ratio estimates for different Sm and Gd isotopic pairs used in 251standardization.

252 Table 4 summarize the purification results of Sm, Eu and Gd from some igneous rocks in 253this study based on HIBA column chromatography of Fig. 1 in this study. Table 4 also 254includes the concentration ratio between $\mathrm{Eu}$ and $\mathrm{Gd}$ from each reference material, which was 255calculated based on the recommend values of the concentration by USGS and GSJ. We also 256measured the REE concentration of all geochemical reference materials and confirmed that 
257our measured REE concentration values were consistent with recommend REE concentration 258values around 5\%.

259 In Table 4, the recovery rate of Eu in most samples were over 99.7\% except JG2. The 260relatively low recovery rate (97. 46 \%) of Eu in JG2 is because, like the rate of Gd impurity 261in the Eu purified fraction, the large concentration difference and overlapped distribution 262coefficient of $\mathrm{Gd}$ and Eu between HIBA and AG50W-X8 resin during column 263chromatography make it very difficult to completely remove the Gd impurity in the Eu 264 fraction.

265 Table 4 apparently seems to indicate that the purification among Eu, Gd and Sm is good in 266all geological samples. However, in JG2, we can notice that Eu concentration in the eluted 267solution is only 3 times higher than that of Gd impurity. In this case, apparently, although the 268separation of Eu seemed very good, when measuring the Eu isotope ratio by MC-ICP-MS, 269the interference of ${ }^{154} \mathrm{Gd}$ or ${ }^{152} \mathrm{Gd}$ to ${ }^{154} \mathrm{Sm}$ or ${ }^{152} \mathrm{Sm}$, respectively, is severe, resulting in false 270Eu isotope. The experimental results related to will be described later. Lee and Tanaka [30] 271proposed a possibility of pseudo-fractionation of Eu isotope ratio from due to interference of 272Gd impurity in the highly fractionated granite and high silica rhyolite. Particularly, like JG2 273in Table 3, the geological samples with extremely lower Eu concentrations, relative to other 274REEs including Sm and Gd, may experience pseudo-fractionation of Eu isotopes due to Sm 275isotope normalization that Sm spike isotopes $\left({ }^{152} \mathrm{Sm}\right.$ and $\left.{ }^{154} \mathrm{Sm}\right)$ for determination of Eu 276isotope ratio are effected by Gd impurity $\left({ }^{152} \mathrm{Gd}\right.$ and $\left.{ }^{154} \mathrm{Gd}\right)$.

277

2783.3. Gd matrix effect during Eu isotope ratio determination in NIST3117a by MC-ICP-MS 279using Sm internal spike

281 Table 5 and Fig. 3 summarize Eu isotope ratios of NIST3117a corrected using various Sm 282isotope pairs in the artificial samples with different degrees of ${ }^{154} \mathrm{Gd}$ matrix effects in 283NIST3117a Eu standard solution. In Figure 2, we confirmed that the Eu isotope ratio of 284NIST3117a without Gd matrix was almost the same regardless of the type of Sm isotope pair 285 for Sm normalization during Eu isotope ratio determination by MC-ICP-MS. However, Table 2865 and Fig. 3 show that Eu isotope ratio of NIST3117a varies with increasing Gd matrix 287regardless of Sm internal isotope pair except ${ }^{147} \mathrm{Sm}-{ }^{149} \mathrm{Sm}$ pair. Particularly, different Sm 288isotope pairs from samples containing more than ca. $1 \%$ of ${ }^{154} \mathrm{Gd}$ interference against ${ }^{154} \mathrm{Sm}$ 
289produced the decreasing trends (a)-(c) or the steady to increasing trends (d)-(e) shown in Fig. 2903. It means that isotope pairs selected for mass bias correction can produce small but 291significant variation in Eu isotope ratio estimates due to Gd matrix. It suggests that Sm 292isotope pairs can specifically create apparent enrichment of ${ }^{151} \mathrm{Eu}$ or ${ }^{153} \mathrm{Eu}$ in natural samples. 293As a result, pseudo-fractionation of Eu isotope ratios occurs for any Sm isotope pair with 294more than ca. $0.1 \%$ of ${ }^{154} \mathrm{Gd}$ interference relative to ${ }^{154} \mathrm{Sm}$.

295 Lee and Tanaka [10] used ${ }^{150} \mathrm{Sm}^{-154} \mathrm{Sm}$ isotope pair for Sm normalization during Eu isotope 296ratio determination by MC-ICP-MS because the Sm mass number of 150 and 154 297conveniently and symmetrically bracket the Eu mass numbers of 151 and 153. And, Figure 2 298revealed that the kind of Sm isotope pair for normalization did not effect in precise and 299accurate determination Eu isotope ratio of ultrapure Eu chemical reagent such as NIST3117a 300determination. Figure 2 also suggests that Eu isotope ratio determined using Sm isotope pair 301such as ${ }^{150} \mathrm{Sm}-{ }^{154} \mathrm{Sm}$ and ${ }^{147} \mathrm{Sm}^{-154} \mathrm{Sm}$ bracketing ${ }^{151} \mathrm{Eu}$ and ${ }^{153} \mathrm{Eu}$ does not show isotope 302 fractionation. However, Table 5 and Fig. 3 seems to suggest that Eu isotope ratio normalized 303by ${ }^{147} \mathrm{Sm}^{-149} \mathrm{Sm}$ was less affected compared to those normalized by other Sm isotope pairs in 304solutions containing Gd matrix.

305

3063.4. Comparison of Eu isotope ratio estimates from geochemical reference rocks 307

308 Previous studies have established Eu isotope ratio results for geological materials and some 309meteorites using MC-ICP-MS [4, 9-11]. Of these, Lee and Tanaka [10] detected a possible 310Eu isotope fractionation in geological samples. Hu et al. [11] also reported Eu isotope ratio 311 variation from meteorite samples and mentioned that Eu isotopic variations correlate with 312mass-dependent Sr isotopic variation. However, in this study, we noticed that there might 313occur pseudo-Eu fractionation due to a trace of Gd matrix in purified Eu fraction from 314geological materials.

315 Tables 6-10 and Figs. 4-6 summarize $\mathrm{Eu}$ isotopic ratio data from four geochemical 316reference rocks measured by a Neptune plus MC-ICP-MS and using various Sm isotope pairs 317 for mass bias correction.

318 Figs. 4-6 clearly indicate that determination method of Eu isotope ratio using Sm isotope 319normalization (C-SSBIN) provide more stable data than that by conventional SSB method 320even though the purified sample include a little of matrix. We can also notice that JG2 
321 (rhombus symbol in Fig. 5) show larger variation in the values of $\delta^{151 / 153} \mathrm{Eu}_{\text {NIST3117a }}$ compared 322 to any other sample in this study. In Table 4, we showed that recovery rate of JG2 is lower 323than any other samples. Eu concentration of JG2 is very low (less than $0.1 \mathrm{ppm}$ ). Even 324though the recovery rate of Eu was more than 97\%, complete purification between Eu and Gd 325was very difficult due to large difference of concentration and similar distribution coefficient 326between two elements (Table 4). Such low concentration of Eu and relatively high Gd 327impurity seems to make dispersion of $\mathrm{Eu}$ isotope ratio determined by MC-ICP-MS. 328Particularly, the variance of Eu isotope ratio showed a tendency to increase in the samples 329subjected to the ${ }^{154} \mathrm{Gd}$ effect of more than $0.1 \%$ for ${ }^{154} \mathrm{Sm}$ when we used $\mathrm{Sm}$ isotope 330normalization to determine for Eu isotope ratio by MC-ICP-MS.

\section{1}

3323.5. Optimal standardization for Eu isotope ratios during MC-ICP-MS analysis 333

334 Previous report [10], we used ${ }^{150} \mathrm{Sm}^{-154} \mathrm{Sm}$ isotope pair for normalization under 7 Faraday 335collecters for Eu isotope ratio determination by MC-ICP-MS. And, we could confine that the 336Eu isotope ratio of pure Eu standard reagent (NIST3117a) measured by MC-ICP-MS show 337almost the same regardless of the type of Sm isotope pair for normalization. However, in 338Figs. 4-6, we can observe that, if the effect of ${ }^{154} \mathrm{Gd}$ on the spike isotope ${ }^{154} \mathrm{Sm}$ is less than $3390.1 \%$, the values of $\delta^{151 / 153} \mathrm{Eu}_{\mathrm{NIST3117a}}$ from each rock reference material determined by Sm 340normalization are almost same regardless of the kind of Sm isotope pair. Particularly, Figs. 43416 suggest that, when we consider Gd matrix effect during Sm normalization, ${ }^{147} \mathrm{Sm}-{ }^{154} \mathrm{Sm}$ or $342^{147} \mathrm{Sm}^{-149} \mathrm{Sm}$ isotope pairs is more profitable in determining precise and accurate Eu isotope 343ratio determination rather than ${ }^{150} \mathrm{Sm}-{ }^{154} \mathrm{Sm}$ and the others.

344

\section{Conclusions}

346

347 We compared Sm and Gd isotopic pairs used as internal standards for measuring Eu 348isotope ratios by MC-ICP-MS in order to find an optimal method for determining precise and 349accurate Eu isotope ratio from various kinds of the geological rocks. The isotope pair ${ }^{147} \mathrm{Sm}-$ $350{ }^{149} \mathrm{Sm},{ }^{147} \mathrm{Sm}-{ }^{154} \mathrm{Sm},{ }^{149} \mathrm{Sm}-{ }^{154} \mathrm{Sm},{ }^{150} \mathrm{Sm}-{ }^{154} \mathrm{Sm}$ and ${ }^{155} \mathrm{Gd}-{ }^{157} \mathrm{Gd}$ were used to perform bracketed 351mass bias correction (C-SSBIN). Our results demonstrated that the ${ }^{147} \mathrm{Sm}^{149} \mathrm{Sm}$ or ${ }^{147} \mathrm{Sm}-$ $352^{154} \mathrm{Sm}$ isotope pair provides the most accurate and precise results (least instrumental mass 
353fractionation) for Eu isotope ratios measured from geological samples whereas ultrapure 354chemical reagent such as NIST3117a Eu standard material do not make any kind of Eu 355isotope ratio variation during operating of MC-ICP-MS using Sm or Gd isotope pairs 356normalization mentioned above. Particularly, high silica geological rocks such highly 357 fractionated granite and rhyolite require higher purity of Eu separation with little of Gd 358impurity with very high degree of Eu recovery because incomplete separation of Eu from Gd 359during sample preparation can bias severe Eu isotope ratio estimates due to interference.

\section{Declaration of interests}

362 The authors declare that they have no known competing financial interests or personal 363relationships that could have influenced or appeared to influence the research reported in this 364paper.

\section{5}

\section{CRediT author statement}

367Seung-Gu Lee: Conceptualization, Methodology, Validation, Formal measurement, 368 Writing-Original Draft, Writing- Reviewing and Editing.

369Tsuyoshi Tanaka: Writing- Reviewing and Editing, Conceptualization.

\section{0}

\section{Acknowledgments}

372 This research was supported by grants from the National Research Foundation of Korea 373(NRF) grant funded by the Korea government (MSIT) (2020R1F1A1075924) and the 374Principal research Fund of the Korea Institute of Geoscience and Mineral Resources 375(GP2020-003). 


\section{References}

377[1] M.J., Drake, The oxidation state of europium as an indicator of oxygen fugacity, 378 Geochim. Cosmochim. Acta 39 (1975) 55-64.

379[2] J.A. Philpotts, Redox estimation from a calculation of Eu2+ and Eu 3+ 380 concentrations in natural phases, Earth Planet. Sci. Lett. 9 (1970) 257-268.

381[3] N. Dauphas, M. Roskosz, E.E. Alp, D.R. Neuville, M.Y. Hu, C.K. Sio, F.L.H. Tissot, 382 J. Zhao, L. Tissandier, M. Médard, C.N. Cordier, Magma redox and structural controls 383 on iron isotope variations in Earth's mantle and crust. Earth Planet. Sci. Lett. 398 384 (2014) 127-140.

385[4] F. Moynier, A. Bouvier, J. Blichert-Toft, P. Telouk, D. Gasperini, F. Albarède., 386 Europium isotopic variations in Allende CAIs and the nature of mass-dependent 387 fractionation in the solar nebula, Geochim Cosmochim Acta 70 (2006) 4287-4294.

388[5] R. Nakada, M. Tanimizu, Y. Takahashi, Difference in the stable isotopic 389 fractionations of $\mathrm{Ce}, \mathrm{Nd}$, and Sm during adsorption on iron and manganese oxides and 390 its interpretation based on their local structures, Geochim. Cosmochim. Acta 121 391 (2013) 105-119.

392[6] T. Ohno, T. Hirata, Determination of mass-dependent isotopic fractionation of cerium and 393 neodymium in geochemical samples by MC-ICP-MS, Anal. Sci. 29 (2013) 47-53.

394[7] S. Wakaki, T. Tanaka, Stable isotope analysis of $\mathrm{Nd}$ by double spike thermal 395 ionization mass spectrometry, Int. J. Mass Spectrom. 323-324 (2012) 45-54.

396[8] S. Wakaki, T. Tanaka, Stable Sm isotopic analysis of terrestrial rock samples by 397 double-spike thermal ionization mass spectrometry, Int. J. Mass Spectrom. 407 (2016) $39822-28$.

399[9] G. G. A. de Carvalho, P.V. Oliveira, L. Yang, Determination of europium isotope 400 ratios in natural waters by MC-ICP-MS, J. Anal. At. Spectrom. 32 (2017) 987-995.

401[10] S-G. Lee, T. Tanaka, Determination of Europium isotopic ratio by multi-collector 402 inductively coupled plasma mass spectrometry using a Sm internal standard, 403 Spectrochim. Acta Part B 156 (2019) 42-50.

404[11] Hu, J.Y., Dauphas, N., Tissot, F.L.H., Yokochi, R., Ireland, T.J., Zhang, J., Davis, 405 A.M., Celsia, F.J., Grossman, L., Charller, B.L.A., Roskosz, M., Alp, E.E., Hu, M.Y. 406 and Zhao, J. (2021) Heating events in the nascent solar system recorded by rare earth 407 element isotopic fractionation in refractory inclusions. Sci. Adv. 7:eabc2962 
408[12] Li, H., Tissot, F.L.H., Lee, S-G., Hyung, E. and Dauphas, N. (2021) Distribution 409 coefficients of REEs, Sr, Y, Ba, Th, and U between $\alpha$-HIBA at pH=4.5 and AG50W410 X8 resin. ACS Ear. Sp. Chem. 5, 55-65.

411[13] S-G. Lee, T. Kim, S. Han, H. C. Kim, H. M. Lee, T. Tanaka, S. R. Lee, J. I. Lee, 412 Effect of Zircon on Rare-Earth Element Determination of Granitoids by ICP-MS, J. 413 Petrol. Soc. Kor. 23 (2014) 337-349.

414[14] S-G. Lee, T. Kim, T. Tanaka, S. R. Lee, J. I. Lee, Effect on the Measurement of 415 Trace Element by Pressure Bomb and Conventional Teflon Vial Methods in the 416 Digestion Technique, J. Petrol. Soc. Kor. 25 (2014) 107-119.

417[15] C.N. Maréchal, P. Télouk, F. Albarède, Precise analysis of copper and zinc isotopic 418 compositions by plasma-source mass spectrometry. Chem. Geol. 156 (1999) 251-273. 419[16] Q.H. Hou, L. Zhou, S. Gao, T. Zhang, L. Feng and L. Yang, Use of Ga for mass 420 bias correction for the accurate determination of copper isotope ratio in the NIST SRM $4213114 \mathrm{Cu}$ standard and geological samples by MC-ICPMS, J. Anal. At. Spectrom. 31 422 (2016) 280-287.

423[17] L. Yang, Accurate and Precise Determination of Isotopic Ratios by MC-ICP-MS: A 424 Review, Mass Spectro. Rev. 28 (2009) 990-1101.

425[18] F. Vanhaecke and P. Degryse, Isotopic Analysis, Wiley-VCH Verlag \& Co. KGaA 426 Boschstr., Weinheim, Germany, 2012.

427[19] A. Makishima, Thermal Ionization Mass Spectrometry (TIMS), Wiley-VCH Verlag 428 \& Co. KGaA Boschstr., Weinheim, Germany, 2016.

429[20] F. Albarède, P. Télouk, J. Blichert-Toft, M. Boyer, A. Agranier, B. Nelson, Precise 430 and accurate isotopic measurements using multiple-collector ICP-MS, Geochim. 431 Cosmochim Acta 68 (2004) 2725-2744.

432[21] H. Hidaka, M. Ebihara, M. Shima, Determination of the Isotopic Compositions of 433 Samarium and Gadolinium by Thermal Ionization Mass Spectrometry, Anal. 434 Chem. 67 (1995) 1437-1441.

435[22] R. Mass, M. T. McCulloch, A search for fossil nuclear reactors in the Alligator 436 River Uranium Field, Australia: Constraints from Sm, Gd and Nd isotopic studies, 437 Chem. Geol. 88 (1990) 301-315. 
438[23] J.C. Dubois, G. Retali and J. Cesario, Isotopic analysis of rare earth elements by 439 total vaporization of samples in thermal ionization mass spectrometry, Int. J. Mass 440 Spectrom. Ion Processes 120 (1992) 163-3177.

441[24] K.E. Jarvis, A.L. Date, R.S. Houk, Handbook of Inductively coupled plasma mass 442 spectrometry, Glasgow, Blackie, 1992.

443[25] K.G. Heumann, S.M. Gallus, G. Rädlinger, J. Vogel, Precision and accuracy in 444 isotopic ratio measurements by plasma source mass spectrometry, J. Anal. At. Spectro. 44513 (1998) 1001-1008.

446[26] H.E. Taylor, R.A. Huff, A. Montaser, Novel applications of ICPMS. In: Montaser 447 A., editor. Inductively coupled plasma mass spectrometry, Inductively coupled plasma 448 mass spectrometry, New York: Wiley-VCH (1998).

449[27] J.S. Becker, Recent developments in isotopic analysis by advanced mass 450 spectrometric techniques, J. Anal. At. Spectrom, 20 (2005) 1173-1184.

451[28] B. Luais, P. Telouk, F. Albarde, Precise and accurate neodymium isotopic 452 measurements by plasma-source mass spectrometry, Geochim. Cosmochim. Acta 61 453 (1997) 4847-4854.

454[29] N. Dauphas, P. E. Janney, R. A. Mendybaev, M. Wadhwa, F. M. Richter, A. M. 455 Davis, M. van Zuilen, R. Hines and C.N. Foley, Chromatographic Separation and 456 Multicollection-ICPMS Analysis of Iron. Investigating Mass-Dependent and 457 Independent Isotope Effects, Anal. Chem. 76 (2004) 5855-5863.

458[30] S-G. Lee, T. Tanaka, Eu isotope fractionation in highly fractionated igneous rocks 459 with large Eu negative anomaly and tetrad effect REE patterns. Geochem. J. (2021) 460 submitted.

461[31] K. Irisawa, T. Hirata, Tungsten isotopic analysis on six geochemical references 462 materials using multiple collector-ICP-mass spectrometry coupled with a rhenium463 external correction technique. J. Anal. At. Spectrom. 21 (2006) 1387-1395.

464[32] M.E. Wieser, J.R. de Laeter, M.D. Varner, Isotopic fractionation studies of 465 molybdenum, Int. J. Mass Spectrom. 265 (2007) 40-48.

466[33] K.J.R. Rosman, P.D.P. Taylor, Isotopic Composition of the Elements 1997, Pure 467 Appl. Chem. 70 (1998) 217-236. 
471Fig. 1. Eu purification result from Sm and Gd. Eu and Gd can be separated almost completely 472 by $0.12 \mathrm{M}$ and 0.09 M 2-hydroxybutric acid (HIBA). The intensity of Eu, Sm and Gd were 473 measured by Neptune Plus MC-ICP-MS.

474

475Fig. 2. Eu isotopic ratios of the NIST3117a measured by MC-ICP-MS using different Sm and 476 Gd internal isotope pairs without Gd or Sm matrix, respectively. $\left({ }^{150} \mathrm{Sm} /{ }^{154} \mathrm{Sm}=0.3244[10\right.$ 477 and 0.325387 [23], ${ }^{147} \mathrm{Sm} /{ }^{154} \mathrm{Sm}=0.6626506,{ }^{147} \mathrm{Sm} /{ }^{149} \mathrm{Sm}=1.0868,{ }^{149} \mathrm{Sm} /{ }^{154} \mathrm{Sm}=$ $4780.609726,{ }^{147} \mathrm{Sm} /{ }^{152} \mathrm{Sm}=0.562992$ and ${ }^{155} \mathrm{Gd} /{ }^{157} \mathrm{Gd}=0.947645$ [23]). For comparison, we 479 calculated the Eu isotope ratio normalized by different value of ${ }^{150} \mathrm{Sm} /{ }^{154} \mathrm{Sm}$. Eu isotope 480 ratio normalized by ${ }^{150} \mathrm{Sm} /{ }^{154} \mathrm{Sm}$ is the same regardless of the value of ${ }^{150} \mathrm{Sm} /{ }^{154} \mathrm{Sm}$.

482Fig. 3. Eu isotope ratio variation (y-axes) of the NIST3117a influenced by ${ }^{152} \mathrm{Gd}$ or ${ }^{154} \mathrm{Gd}$ 483 interference or matrix effects (x-axes) for different Sm isotope pairs used to correct for 484 mass bias during MC-ICP-MS analysis. C-SSBIN: (a) ${ }^{150} \mathrm{Sm} /{ }^{154} \mathrm{Sm}$, (b) ${ }^{147} \mathrm{Sm} /{ }^{154} \mathrm{Sm}$, (c) $485{ }^{149} \mathrm{Sm} /{ }^{154} \mathrm{Sm}$, (d) ${ }^{149} \mathrm{Sm} /{ }^{154} \mathrm{Sm}$, (e) ${ }^{147} \mathrm{Sm} /{ }^{152} \mathrm{Sm}$, (f) SSB without Sm normalization. 486

487Fig. 4. . Eu isotope ratio variation (y-axes) of three basalts (BCR2 and BIR2 from USGS; JB3 488 from GSJ) influenced by ${ }^{152} \mathrm{Gd}$ or ${ }^{154} \mathrm{Gd}$ interference or matrix effects (x-axes) for different 489 Sm isotope pairs used to correct for mass bias during MC-ICP-MS analysis. The Eu 490 isotope ratio was determined using (a) ${ }^{150} \mathrm{Sm} /{ }^{154} \mathrm{Sm}=0.325396$, (b) ${ }^{147} \mathrm{Sm} /{ }^{154} \mathrm{Sm}=$ 491 0.6626506, (c) ${ }^{147} \mathrm{Sm} /{ }^{149} \mathrm{Sm}=1.0868$, (d) ${ }^{149} \mathrm{Sm} /{ }^{154} \mathrm{Sm}=0.609726$, (e) ${ }^{147} \mathrm{Sm} /{ }^{152} \mathrm{Sm}=$ 4920.562992 [23] (f) standard-sample-bracketing method without normalization.

494Fig. 5. Eu isotope ratio variation (y-axes) of three granites (GSP2 from USGS; JG1a and JG2 495 from GSJ) influenced by ${ }^{152} \mathrm{Gd}$ or ${ }^{154} \mathrm{Gd}$ interference or matrix effects (x-axes) for different 496 Sm isotope pairs used to correct for mass bias during MC-ICP-MS analysis. The Eu 497 isotope ratio was determined using (a) ${ }^{150} \mathrm{Sm} /{ }^{154} \mathrm{Sm}=0.325396$, (b) ${ }^{147} \mathrm{Sm} /{ }^{154} \mathrm{Sm}=$ 498 0.6626506, (c) ${ }^{147} \mathrm{Sm} /{ }^{149} \mathrm{Sm}=1.0868$, (d) ${ }^{149} \mathrm{Sm} /{ }^{154} \mathrm{Sm}=0.609726$, (e) ${ }^{147} \mathrm{Sm} /{ }^{152} \mathrm{Sm}=$ 4990.562992 [23] (f) standard-sample-bracketing method without normalization. 500Fig. 6. Eu isotope ratio variation (y-axes) of gabbro (JGb2, GSJ) and rhyolite (RGM2, USGS) 501 influenced by ${ }^{152} \mathrm{Gd}$ or ${ }^{154} \mathrm{Gd}$ interference or matrix effects (x-axes) for different Sm isotope 
502 pairs used to correct for mass bias during MC-ICP-MS analysis. The Eu isotope ratio was 503 determined using (a) ${ }^{150} \mathrm{Sm} /{ }^{154} \mathrm{Sm}=0.325396$, (b) ${ }^{147} \mathrm{Sm} /{ }^{154} \mathrm{Sm}=0.6626506$, (c) ${ }^{147} \mathrm{Sm} /{ }^{149} \mathrm{Sm}$ $504=1.0868$, (d) ${ }^{149} \mathrm{Sm} /{ }^{154} \mathrm{Sm}=0.609726$, (e) ${ }^{147} \mathrm{Sm} /{ }^{152} \mathrm{Sm}=0.562992$ [23] (f) standard505 sample-bracketing method without normalization.

506

507 